\title{
PRINCIPAIS CAUSAS DE DESCARTE EM BÚFALOS ABATIDOS NO ESTADO DO RIO GRANDE DO SUL, BRASIL
}

Recebido: $25 / 03 / 2015$

\author{
MACHADO, Gilmar Batista ${ }^{1}$; \\ MOURA, Sandra Vieira de ${ }^{2}$; \\ FORTES, Tanise Pacheco ${ }^{2}$; \\ FACIN, Diego Viedo ${ }^{3}$; \\ LANSINI, Valmor ${ }^{3}$; \\ SILVA, Éverton Fagonde da ${ }^{4}$.
}

${ }^{1}$ Doutorando do Programa de Pós-Graduação em Veterinária, UFPEL; ${ }^{2}$ Doutoranda do Programa de PósGraduação em Veterinária, UFPEL; ${ }^{3}$ Coordenadoria de Inspeção de Produtos de Origem Animal (CISPOA), Pelotas, RS; ${ }^{4}$ Médico Veterinário, Professor Doutor, Faculdade de Veterinária, UFPEL.

\section{RESUMO}

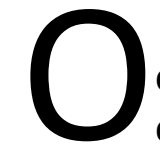

Brasil possui um rebanho de búfalos com aproximadamente um milhão cento e cinquenta mil cabeças. No estado do Rio Grande do Sul (RS), a bubalinocultura de corte vem crescendo nos últimos anos, principalmente pela precocidade dos animais e pela qualidade da carne. A inspeção post-mortem de órgãos e carcaças é fundamental no processo de triagem de animais e da carne para o consumo humano. Assim, o presente trabalho objetivou analisar e discutir as principais causas de condenação de órgãos e carcaças encontradas durante a inspeção post-mortem de bubalinos em abatedouros sob inspeção estadual no RS, no período entre janeiro e dezembro de 2010. Foram inspecionados 5.614 bubalinos pelas técnicas de exame visual, palpação e incisão de órgãos, realizadas conforme a legislação brasileira. A principal causa de condenação de órgãos foi a congestão pulmonar $(10,44 \%)$, seguida por nefrite $(8,48 \%)$. Entre as doenças infecciosas e parasitárias, a mais frequente foi fasciolose $(6,16 \%)$, seguida pela hidatidose $(4,11 \%)$, actinobacilose $(0,50 \%)$, cisticercose $(0,43 \%)$ e tuberculose $(0,02 \%)$. Após análise dos resultados, pode-se concluir que fasciolose e hidatidose são importantes causas de condenação no RS. Entretanto, outras causas de condenação devem ser consideradas e estudadas.

Palavras-chave: Carne. Carcaças. Zoonoses. Inspeção. Saúde Pública. 


\section{INTRODUÇÃO}

Atualmente, o Brasil possui um rebanho de aproximadamente um milhão cento e cinquenta mil cabeças de bubalinos (MAPA, 2016). Nas últimas décadas, a bubalinocultura ganhou destaque no cenário nacional, deixando de ser apenas uma alternativa para a ocupação de terras impróprias para a criação de bovinos, e passando a ser uma opção economicamente rentável. Com isso, a preocupação com o manejo sanitário tem aumentado consideravelmente, pois aspectos epidemiológicos, clínicos e patológicos de enfermidades relacionadas a essa espécie, ainda são pouco estudados no país (SILVA et al., 2014).

Os bubalinos são considerados animais versáteis, sendo explorados de forma sustentável até mesmo em ambientes inóspitos para outras atividades pecuárias (DAMÉ, 2009). Desde a última década, a bubalinocultura de corte brasileira vem ocupando um importante espaço no cenário nacional, principalmente em razão de sua rusticidade, pois podem apresentar melhor produtividade que os bovinos em ambientes onde o solo e as pastagens possuem qualidade inferior (VAZ et al., 2003). Em sistemas de confinamento, os quais utilizam dietas com rações balanceadas, pode ser observado um maior ganho diário de peso e uma redução da idade de abate, refletindo positivamente sobre as características qualitativas e quantitativas da carcaça e da carne de bubalinos em comparação aos bovinos, como na região do dianteiro, serrote e costilhar (VAZ et al., 2003). Além disso, o investimento no melhoramento genético dos búfalos brasileiros pode resultar em animais ainda mais precoces e carcaças com melhores rendimentos (JORGE, 2005).

Apesar das semelhanças zootécnicas entre bubalinos e bovinos, existem algumas peculiaridades que devem ser levadas em consideração quanto à sanidade dos animais. De uma maneira geral, os bubalinos podem ser acometidos pelas mesmas enfermidades que os bovinos, porém, os búfalos podem apresentar maior resistência a algumas enfermidades comuns aos demais ruminantes, como por exemplo, a intoxicação por Senecio spp. (DAMÉ, 2009). No entanto, durante a inspeção post-mortem de órgãos e carcaças de bubalinos, estudos demonstraram a existência de lesões típicas de doenças infecciosas e parasitárias que acometem bovinos, como tuberculose, cisticercose, fasciolose e hidatidose, as quais são importantes zoonoses e causam graves problemas de saúde pública no Brasil (FREITAS et al., 
1997). Além disso, inúmeras outras patologias podem estar presentes, como as tecnopatias de abate, que estão relacionadas com falhas tecnológicas e de manejo durante esse processo, as quais podem acarretar prejuízos econômicos devido ao descarte de órgãos e carcaças. Nesse contexto, o objetivo do presente trabalho foi descrever as principais causas de condenação de órgãos e carcaças de bubalinos, sob inspeção estadual, no estado do Rio Grande do Sul no ano de 2010.

\section{MATERIAL E MÉTODOS}

Neste estudo, analisou-se as informações disponibilizadas pelo Departamento de Defesa Agropecuária (DDA), Divisão de Inspeção de Produtos de Origem Animal (DIPOA), da Secretaria da Agricultura, Pecuária e Irrigação do Rio Grande do Sul (SEAPI/RS), sobre as lesões encontradas durante o abate de bubalinos, em abatedouros sob fiscalização estadual, no período compreendido entre 01 de janeiro e 31 de dezembro de 2010. As informações foram obtidas das 19 supervisões regionais do DDA, as quais englobam todos os 496 municípios do Rio Grande do Sul. São elas: Alegrete, Bagé, Caxias do Sul, Cruz Alta, Erechim, Estrela, ljuí, Lagoa Vermelha, Osório, Palmeira das Missões, Passo Fundo, Pelotas, Porto Alegre, Rio Pardo, Santa Maria, Santa Rosa, São Luiz Gonzaga, Soledade e Uruguaiana.

Durante o período do estudo foram abatidos 5.614 bubalinos sob inspeção de fiscais do DDA/SEAPI/RS. Os procedimentos de rotina foram realizados de acordo com o Regulamento de Inspeção Industrial e Sanitária de Produtos de Origem Animal (BRASIL, 1997), utilizando as técnicas de exame visual da carcaça e órgãos, palpação e incisão de órgãos, sendo analisados os motivos de condenação parcial ou total da carcaça, cabeça, língua, rins, coração, pulmões, fígado, baço, esôfago, retículo, rúmen, omaso, abomaso e intestino dos bubalinos abatidos. As lesões encontradas nos frigoríficos foram registradas e encaminhadas mensalmente à Seção de Epidemiologia e Estatística (SEE/DDA/SEAPI/RS).

\section{RESULTADOS E DISCUSSÃO}

Do total dos 5.614 bubalinos abatidos durante o ano de 2010 nas regionais do DDA/SEAPI/RS, 346 (6,16\%) animais apresentaram lesões de fasciolose, 231 (4,11\%) animais com lesões de hidatidose, 28 (0,50\%) com lesões de actinobacilose e 24 (0,43\%) animais apresentaram lesões de cisticercose. Em relação a tuberculose, um (0,02\%) animal 
apresentou lesões disseminadas em vários órgãos e na carcaça, causando sua condenação total. Na Tabela 1, pode-se observar, além das doenças identificadas, outras patologias relacionadas aos sistemas renal, hepático, cardíaco e por outras causas relevantes. As alterações nos sistemas renal e pulmonar respondem pela maioria das patologias encontradas nas vísceras inspecionadas (41,94\%). O destino das carcaças quando acometidas por lesões atribuíveis às zoonoses, foi conduzido de acordo com a legislação vigente (BRASIL, 1997).

Tabela 1 - Causas de condenação durante o abate de 5.614 búfalos, sob inspeção estadual, no RS em 2010.

\begin{tabular}{|c|c|c|}
\hline Causa de condenação & Animais afetados (n) & $\%$ \\
\hline \multicolumn{3}{|l|}{ Doenças } \\
\hline Actinobacilose & 28 & 0,50 \\
\hline Cisticercose & 24 & 0,43 \\
\hline Fasciolose & 346 & 6,16 \\
\hline Hidatidose & 231 & 4,11 \\
\hline Tuberculose & 1 & 0,02 \\
\hline \multicolumn{3}{|l|}{ Alterações renais } \\
\hline Cálculo & 18 & 0,32 \\
\hline Enfarte & 23 & 0,41 \\
\hline Isquemia & 191 & 3,40 \\
\hline Litíase & 5 & 0,09 \\
\hline Nefrite & 476 & 8,48 \\
\hline Quisto urinário & 132 & 2,35 \\
\hline Uronefrose & 116 & 2,07 \\
\hline \multicolumn{3}{|l|}{ Alterações hepáticas } \\
\hline Cirrose & 73 & 1,30 \\
\hline Enfarte & 3 & 0,05 \\
\hline Esteatose & 33 & 0,59 \\
\hline Peri-hepatite & 192 & 3,42 \\
\hline Telangiectasia & 103 & 1,83 \\
\hline \multicolumn{3}{|l|}{ Alteração Cardíaca } \\
\hline Pericardite & 89 & 1,59 \\
\hline \multicolumn{3}{|l|}{ Alterações Pulmonares } \\
\hline Congestão & 586 & 10,44 \\
\hline Aspiração de sangue & 315 & 5,61 \\
\hline Aspiração do conteúdo ruminal & 248 & 4,42 \\
\hline Enfisema & 162 & 2,89 \\
\hline Abscesso & 82 & 1,46 \\
\hline \multicolumn{3}{|l|}{ Outras alterações } \\
\hline Contaminação da carcaça & 353 & 6,29 \\
\hline Contusão/fratura & 4 & 0,07 \\
\hline
\end{tabular}


De acordo com os dados disponibilizados pelo DDA/SEAPI/RS, relativos a uma série histórica sobre o abate de búfalos no RS, no período entre 2001 e 2009, observou-se um aumento na frequência da hidatidose, que na média desses anos foi de 2,78\% e em 2010 atingiu um valor médio de $4,11 \%$, assim como a cisticercose, que passou de 0,31 para $0,43 \%$ no mesmo período. A fasciolose apresentou uma diminuição, entre os anos de 2001 e 2009 a média de condenações que se encontrava no patamar de 8,08\% passou para 6,16\% em 2010 , do mesmo modo, a tuberculose passou de 0,12\% para 0,02\% (SANTOS et al., 2010).

No RS, a prevalência da hidatidose é considerada elevada em comparação aos outros estados do Brasil (SANTOS et al., 2010). A frequência é maior em propriedades onde os animais como bovinos, bubalinos e ovinos são criados juntos. Nessas condições, os ovinos (hospedeiros intermediários) tornam-se fontes de infecção para os cães (hospedeiros definitivos), os quais contaminam as pastagens, pois frequentemente os ovinos são abatidos na propriedade rural, facilitando o acesso dos cães às vísceras infectadas com as larvas presentes nos cistos hidáticos, em seguida esses cães passam a infectar com os ovos da tênia Echinococcus granulosos as áreas de pastoreio dos ruminantes (CRINGOLI et al., 2007; SCALA et al., 2004).

A prevalência média da cisticercose no RS, em bovinos abatidos sob inspeção estadual entre 2001 e 2009 foi de 1,87\%, a de búfalos foi de 0,31\%, neste estudo a média foi de 0,43\%. Essa enfermidade é objeto de campanha educativa específica, por parte das Secretarias da Saúde e da Agricultura, nos municípios e propriedades do estado e tem apresentado uma curva de tendência descendente (SANTOS et al., 2010). A doença relaciona-se a pequenas propriedades rurais com deficientes condições de saneamento básico e educação sanitária, onde homens e animais mantêm um contato próximo (ALMEIDA et al., 2002; ESTEVES et al., 2005).

Outra enfermidade com alta prevalência no RS é a fasciolose, especialmente no sul e sudeste do estado, onde é endêmica, zonas tradicionais de cultivo de arroz irrigado, onde a presença da Fasciola hepatica e do seu hospedeiro intermediário (moluscos do gênero Lymnaea) encontram condições propícias de temperatura e umidade para o seu desenvolvimento (MÜLLER, 2007). 
No caso da tuberculose, a enfermidade possui uma relação estreita com a criação de bovinos e bubalinos leiteiros. A doença em búfalos declinou, comparando a média de $0,12 \%$ entre os anos de 2001 e 2009 e o índice de 0,02\% em 2010, provavelmente devido ao Programa Nacional de Controle e Erradicação da Brucelose e Tuberculose Animal (PNCEBT) instituído pelo Ministério da Agricultura, Pecuária e Abastecimento em 2001, em todo território nacional (SANTOS et al., 2010).

Alguns estudos sobre alterações renais encontradas em bovinos abatidos nos estados do Espírito Santo, Goiás, Rio de Janeiro, São Paulo e Distrito Federal, assim como neste estudo, mostraram que as principais causas de condenações de rins estão relacionadas as seguintes patologias: nefrite, quisto urinário, isquemia e uronefrose (BAPTISTA, 2008; OLIVEIRA et al., 2005; PALMA, 2013; SALGADO et al., 2004).

A telangiectasia, dilatação dos capilares sinusóides do fígado, é particularmente comum no gado bovino, não tendo significado clínico (THOMSON, 1998). Trabalhos envolvendo o estudo dessa alteração em bovinos abatidos citam taxas de condenações que vão desde 1,60\% (VECHIATO, 2009) a 41,61\% (BONESI et al., 2003).

Vechiato (2009) observou uma alta frequência de abscessos hepáticos, em um frigorífico paulista, em bovinos procedentes de confinamentos, e os descreve, macroscopicamente, como processos inflamatórios circunscritos, delimitados, com formação de cápsula de tecido conjuntivo fibroso, causados por bactérias.

Nos estudos realizados por Ribeiro (2011) a peri-hepatite foi à alteração anatomopatológica mais evidenciada, sendo encontrada em $82,45 \%$ dos fígados estudados, valores maiores que os encontrados por Silva (1996), que constatou uma taxa de 79,07\% em bubalinos oriundos da Ilha de Marajó. De acordo com Freitas et al. (1995), ao estudarem essa patologia na espécie bubalina, isolaram como agente etiológico a Chlamydia psittaci.

Estudando alterações pulmonares de búfalos abatidos no estado do Pará, Silva (2011) constatou enfisema em $36,37 \%$, congestão em 19,51\%, aspiração de conteúdo ruminal em $18,17 \%$ e aspiração de sangue em $16,50 \%$ dos casos, valores bem mais elevados do que os observados neste estudo. Segundo Wilson (1970), essas alterações acontecem de modo 
acidental durante o processo de abate, já Roça et al. (2001), relacionam essas causas com ineficiência na insensibilização, etapa que antecede a sangria.

Existem poucos estudos disponíveis sobre as causas de condenação durante o abate de bubalinos. Sendo assim, o presente estudo contribuiu para aumentar o conhecimento da ocorrência de enfermidades e alterações passíveis de identificação durante a inspeção postmortem de bubalinos no estado do Rio Grande do Sul.

\section{CONCLUSÃO}

Os resultados encontrados permitem concluir que as zoonoses fasciolose e hidatidose são importantes causas de condenação de órgãos de bubalinos, em abatedouros sob inspeção estadual, no estado do Rio Grande do Sul. Outras causas de condenação mostraram índices elevados, entre elas, aspiração do conteúdo ruminal, aspiração de sangue e contaminação da carcaça, passíveis de redução através de treinamento de pessoal e adequação da estrutura e dos equipamentos para o abate dessa espécie.

\section{MAIN CAUSES OF DISPOSAL OF BUFFALOES SLAUGHTERED IN THE STATE OF RIO GRANDE DO SUL, BRAZIL}

\section{ABSTRACT}

B razil has a 1.150 million buffalo population approximately. In Rio Grande do Sul (RS), the beef buffalo exploitation has increased in the recent years, mainly because of the precocity of the animals and the high quality of meat. Post-mortem inspection of organs and carcasses is fundamental in the process of screening animals and meat for human consumption. Thus, this study aimed to analyze and discuss the main causes of condemnation of organs and carcasses found during the post mortem inspection of buffaloes slaughtered under state inspection between January and December 2010. A total of 5614 buffaloes were slaughtered and post-mortem routine procedures were carried out by viewing, palpation and incision in accordance with Brazilian legislation. The main cause of viscera condemnation was lung congestion (10.44\%), followed by nephritis (8.48\%). Among the infectious and parasitic diseases, the most frequent was the fascioliasis $(6.16 \%)$, followed by hydatidosis $(4.11 \%)$, actinobacillosis $(0.50 \%)$, cysticercosis $(0.43 \%)$, and tuberculosis $(0.02 \%)$. According to the results, fascioliasis and hydatidosis are important 
causes of condemnation in the RS. However, other causes of condemnation should be considered and studied.

Keywords: Meat. Carcasses. Zoonosis. Inspection. Public Health.

\section{PRINCIPALES CAUSAS DE DESCARTE EN BUFFALOS SACRIFICADOS EN RIO GRANDE DO SUL, BRASIL}

\section{RESUMEN}

B rasil tiene una manada de búfalos con aproximadamente un millón ciento cincuenta mil cabezas. En Rio Grande do Sul (RS), bubalinocultura de carne ha ido creciendo en los últimos años, principalmente debido a la precocidad de los animales y calidad de la carne. La inspección veterinaria post-mortem de órganos y carcasas de animales es esencial en el proceso de selección de la carne para el consumo humano. Así, este estudio tuvo como objetivo analizar y discutir las principales causas de la condena de los órganos y cadáveres encontrados en la inspección post-mortem de los búfalos de inspección estatal de los mataderos en RS, entre enero y diciembre de 2010. Hubo 5.614 búfalos inspeccionados utilizando las técnicas de examen visual, palpación e incisión de órganos, realizada según la ley brasileña. La principal causa de la condena de los órganos fue la congestión de los pulmones $(10,44 \%)$, seguido de la nefritis $(8,48 \%)$. Entre las enfermedades infecciosas y parasitarias, la más frecuente fue fasciolasis $(6,16 \%)$, seguido de la hidatidosis $(4,11 \%)$, actinobacilosis $(0,50 \%)$, la cisticercosis $(0,43 \%)$ y la tuberculosis $(0,02 \%)$. Tras analizar los resultados, se puede concluir que la fasciolasis y la hidatidosis son causas importantes de la condena en RS. Sin embargo, otras causas de la condena deben ser consideradas y estudiadas.

Palabras clave: Carne. Carcasas. Zoonosis. Inspección. Salud pública.

\section{AGRADECIMENTOS}

Os autores agradecem a Coordenação de Aperfeiçoamento de Pessoal de Nível Superior (CAPES) pelo apoio financeiro para execução do projeto e pelas bolsas de mestrado de G. B. M. e T. P. F., e a de doutorado de S. V. M., e também ao Conselho Nacional de Desenvolvimento Tecnológico (CNPq), processo no 309163/2012-9. 


\section{REFERÊNCIAS}

ALMEIDA, L. P.; MOREIRA, M. D.; REIS, D. O.; SANTOS, W. L. M. Cisticercose bovina: um estudo comparativo entre animais abatidos em frigoríficos com serviço de Inspeção Federal e com Inspeção Municipal. Revista Higiene Alimentar, v. 16, p. 51-55, 2002.

BAPTISTA, A. T. Quantificação das condenações de vísceras de Bovinos, em 2007, nos matadouros frigoríficos do Estado do Espírito Santo, registrados no Serviço de Inspeção Estadual. Vitória: UCB, 2008, 22p. Monografia (Especialização), Universidade Católica de Brasília, 2008.

BONESI, G. L.; SCALONE, B. C. V.; OKANO, W.; ROSA, A. Lesões hepáticas em bovinos abatidos em matadouro-frigorífico. Higiene Alimentar, v. 17, n. 106, p. 78-83, 2003.

BRASIL. Regulamento de Inspeção Industrial e Sanitária de Produtos de Origem Animal. Aprovado pelo decreto $\mathrm{n}$ 0 30.691, de 29/03/52, alterado pelos decretos $\mathrm{n}$ - 1.255 , de 25/06/62, no 1.236, de 02/09/94, no 1.812, de 08/02/96 e № 2.244, de 04/06/97. Diário Oficial da União, Seção I, p. 11555-11558, 1997.

CRINGOLI, G.; RINALDI, L.; MUSELLA, V.; VENEZIANO, V.; MAURELLI, M. P.; DI PIETRO, F.; FRISIELLO, M.; DI PIETRO, S. Geo-referencing livestock farms as tool for studying cystic echinococcosis epidemiology in cattle and water buffaloes from southern Italy. Geospatial Health, v. 2, p. 105-111, 2007.

DAMÉ, M. C. F. Considerações sobre algumas doenças infecciosas, tóxicas e congênitas de interesse à bubalinocultura do extremo sul do país, 2009. Disponível em:

<http://www.cpact.embrapa.br/publicacoes/download/documentos/documento_263.pdf>. Acessado em: 04 de maio de 2012.

ESTEVES, F. M.; SILVA-VERGARA, M. L.; CARVALHO, A. C. F. B. Inquérito epidemiológico sobre teníase em população do programa de saúde da família no município Uberaba-MG. Revista da Sociedade Brasileira de Medicina Tropical, v. 38, n. 6, p. 530-531, 2005.

FREITAS, J. A.; MACHADO, R. D.; NAKAUTH, C.; NORONHA, S. L. B; RAMOS, O. S. Caracterização da cepa Clamydia psittaci oriunda de polisserosite de bubalinos. Arquivos do Instituto Biológico, v. 62, p. 53-58, 1995.

FREITAS, J. A.; BARROS, M. J. C.; NASCIMENTO, J. A. C. Alterações similares à tuberculose no abate de bovinos e bubalinos e no nível de consumo. FCAP: Informe Técnico, n. 23, p. 6-23, 1997.

JORGE, A. M. Produção de carne bubalina. Revista Brasileira de Reprodução Animal, v. 29, n. 2, p. 84-95, 2005. 
MAPA - Ministério da Agricultura, Pecuária e Abastecimento. Bovinos e Bubalinos. Disponível em: <http://www.agricultura.gov.br/animal/especies/bovinos-e-bubalinos>. Acessado em 04 de abril de 2016.

MÜLLER, G. Fasciolose. In: RIET-CORREA, F.; SCHILD, A. L.; LEMOS, R. A. A.; BORGES, J. R. J. (Eds). Doenças de Ruminantes e Equinos. Santa Maria: Palloti, 4. Ed. 2007, 722p.

OLIVEIRA, I.; POMBO, C. R.; SILVA, T. P.; COSTA, L. A. S.; BARROS, S. J.; COSTA, R. S. Ocorrência de condenações nas linhas de inspeção em matadouros frigoríficos de bovinos no município de Campos dos Goytacazes-RJ. In: CONGRESSO LATINO-AMERICANO DE HIGIENISTAS DE ALIMENTOS, 2, 2005, Búzios. Resumos. Búzios: CLAHA, 2005.

PALMA, J. M. Principais lesões em carcaças e órgãos de bovinos oriundos de frigoríficos no Distrito Federal e Goiás. Brasília, UNB, 2013, 26p. Monografia (Graduação em Medicina Veterinária), Faculdade de Agronomia e Medicina Veterinária, Universidade de Brasília, 2013.

RIBEIRO, D. B. Estudo anatomo e histopatológico de fígados bovinos e bubalinos criados na Ilha de Marajó, estado do Pará, condenados pelo serviço de inspeção estadual. Rio de Janeiro: UFF, 2011, 63p. Tese (Doutorado em Higiene Veterinária e Processamento Tecnológico de Produto de Origem Animal), Programa de Pós-Graduação em Medicina Veterinária, Universidade Federal Fluminense, 2011.

ROÇA, R. O.; PADOVANI, C. R.; FILIPI, M. C.; SCHWACH, E.; BIONDI, G. F. Efeito dos métodos de Abate de bovinos, na eficiência da Sangria. Revista Ciência e Tecnologia de alimentos, $v$. 21, n. 2, p. 244-248, 2001.

SALGADO, R. L.; MILAR, P. R.; BOM, L. C.; CAMARGO, G. L.; SILVA, T. J. P. Ocorrência de condenações e aproveitamento condicional no abate de bovinos em um matadouro frigorífico no estado de São Paulo. Arquivos de Ciências Veterinárias e Zoologia da Unipar. Paraná, v. 7, p. 70-71, 2004.

SANTOS, D. V.; ESPÍRITO SANTO, M. C. B.; DOMINGUES, E. H.; JÚNIOR, I. K.; FACIN, D. V.; VIDOR, A. C. Análise das principais lesões encontradas nos abatedouros registrados na CISPOA. Informativo técnico-DPA, v. 4, p. 01-07, 2010.

SCALA, A.; VARCASIA, A.; GARIPPA, G. Cystic echinococcosis in Sardinia: the current role of sheep. Parasitologia, v. 46, p. 397-400, 2004.

SILVA, J. B.; LOPES, C. T. A.; SOUZA, M. G. S.; GIBSON, A. F. B.; VINHOTE, W. M. S.; FONSECA, A. H.; ARAÚJO, F. R.; BARBOSA-NETO, J. D. Detecção sorológica e molecular de Anaplasma marginale em búfalos na llha de Marajó, Pará. Pesquisa Veterinária Brasileira, v. 34, n. 1, p. 11-14, 2014. 
SILVA, R. A. G. Morfologia das alterações hepáticas em bubalinos (Bubalus bubalis lin) abatidos para consumo em Belém-Pará, com ênfase à periepatite. Niterói, UFF, 1996, 37p. Dissertação (Mestrado em Higiene Veterinária e Processamento Tecnológico de Produtos de Origem Animal), Universidade Federal Fluminense, 1996.

SILVA, R. N. S. Alterações pulmonares de interesse para a inspeção sanitária em bubalinos e bovinos abatidos no estado do Pará. Niterói, UFF, 2011, 62p. Tese (Doutorado em Higiene Veterinária e Processamento Tecnológico de Produtos de Origem Animal), Universidade Federal Fluminense, 2011.

THOMSON, R. G. Patologia veterinária especial. São Paulo: Manole, 1998.

VAZ, F. N.; RESTLE, J.; BRONDANI, I. L.; PACHECO, P. S. Estudo da carcaça e da carne de bubalinos Mediterrâneo terminados em confinamento com diferentes fontes de volumoso. Revista Brasileira de Zootecnia, v. 32, p. 393-404, 2003.

VECHIATO, T. A. F. Estudo retrospectivo e prospectivo da presença de abscessos hepáticos em bovinos abatidos em um frigorífico paulista. São Paulo: USP, 2009. Dissertação (Mestrado em Medicina Veterinária), Faculdade de Medicina Veterinária e Zootecnia, Universidade de São Paulo, 2009.

WILSON, A. Inspeccion Prática de la Carne. Zaragoza: Acribia, p. 138-139, 1970. 